\title{
Value of Dynamic Contrast-Enhanced (DCE) MR Imaging in Peripheral Lesions in PI-RADS-4 Patients
}

\section{Stellenwert der dynamischen kontrastmittelgestützten MR-Bildgebung in peripheren Läsionen bei PI-RADS-4-Patienten}

Authors

Tim Ullrich ${ }^{1}$, Michael Quentin ${ }^{1}$, Christian Arsov², Nina Laqua', Daniel Abrar ${ }^{1}$, Andreas Hiester ${ }^{2}$, Peter Albers ${ }^{2}$, Gerald Antoch ${ }^{1}$, Lars Schimmöller ${ }^{1}$

Affiliations

1 University Dusseldorf, Medical Faculty, Department of Diagnostic and Interventional Radiology, Dusseldorf, Germany

2 University Dusseldorf, Medical Faculty, Department of Urology, Dusseldorf, Germany

Key words prostate cancer, magnetic resonance imaging, contrast media, image-guided biopsy

received 12.04 .2019

accepted 23.09.2019

Bibliography

DOI https://doi.org/10.1055/a-1020-4026

Published online: 17.10.2019

Fortschr Röntgenstr 2020; 192: 441-447

(c) Georg Thieme Verlag KG, Stuttgart · New York

ISSN 1438-9029

Correspondence

Priv.-Doz. Dr. Lars Schimmöller

Institut für Diagnostische und Interventionelle Radiologie, Universitätsklinikum Düsseldorf, Moorenstraße 5,

40225 Düsseldorf, Germany

Tel.: ++ 49/211/8117754

Fax: $++49 / 211 / 8116145$

lars.schimmoeller@med.uni-duesseldorf.de

\section{ZUSAMMENFASSUNG}

Ziel Ziel der Studie war die Evaluation des Mehrwerts der dynamischen kontrastmittelgestützten Sequenzen (DCE) in der multiparametrischen MRT (mp-MRT) bezüglich der Prostatakarzinomdetektion in einer großen Kohorte von Patienten mit PI-RADS-4-Befund.

Material und Methoden 193 Patienten mit einer MR-Gesamtklassifikation von PI-RADS 4 nach mp-MRT der Prostata bei 3 T (T2WI, DWI, DCE) und anschließend kombiniert gezielter plus systematischer Biopsie als Referenzstandard wurden retrospektiv in diese unizentrische Kohortenstudie eingeschlossen. Es wurde die Prostatakarzinomdetektion mit und ohne die Hilfe kontrastmittelgestützter Sequenzen verglichen.
Ergebnisse Die Gesamtkarzinomdetektionsrate in den PI-RADS-4-Patienten war $62 \%$ (119/193). Ohne Einschluss von Läsionen, die mithilfe der DCE von Kategorie PI-RADS 3 auf PI-RADS 4 aufgewertet wurden, lag die Detektionsrate bei $52 \%$ (101/193). Mit DCE wurden $48 \%$ (92/193) klinisch signifikante Prostatakarzinome (Gleason-Score $\geq 3+4=7$ ) detektiert und $40 \%$ (78/193) ohne DCE-Verwendung. 38 der 193 Patienten (20\%) hatten periphere Läsionen, die durch auffällige Kontrastmittelanreicherung von PI-RADS Kategorie 3 auf 4 aufgewertet wurden. Von diesen 38 Patienten hatten 18 ein Prostatakarzinom, einschließlich 14 mit einem klinisch signifikanten Karzinom. Somit wurden $15 \%$ (18/119) mit jeglichem Prostatakarzinom und 15\% (14/92) mit einem klinisch relevanten Karzinom nur durch zusätzliche Informationen aus kontrastmittelgestützten Sequenzen detektiert.

Schlussfolgerung Kontrastmittelgestützte, dynamische Sequenzen können eine zu niedrige PI-RADS-Fehlklassifizierung einer relevanten Anzahl von Prostatakarzinomen verhindern und die Detektionsraten bei PI-RADS-4-Patienten verbessern. Die aktuellen PI-RADS-Kriterien zur Aufwertung von PI-RADS3-Läsionen in die Gesamtkategorie 4 durch auffällige Kontrastmittelanreicherung scheinen sinnvoll.

\section{Kernaussagen:}

- $20 \%$ der PI-RADS-4-Patienten wurden durch auffällige, periphere Kontrastmittelanreicherung aus Kategorie 3 aufgewertet.

- Klinisch signifikante Prostatakarzinome wurden in fast $40 \%$ der aufgewerteten, peripheren PI-RADS-3-Läsionen gefunden.

- $15 \%$ aller signifikanten Karzinome bei PI-RADS-4-Patienten wurden in durch DCE aufgewerteten Läsionen detektiert.

- In 7\% aller PI-RADS-4-Befunde wären klinisch signifikante Prostatakarzinome ohne DCE unterschätzt worden.

\section{ABSTRACT}

Objective To assess the impact of dynamic contrast-enhanced imaging (DCE) in mp-MRI on prostate cancer (PCa) detection in a large patient cohort assigned to PI-RADS category 4. 
Method This retrospective, single center cohort study includes 193 consecutive patients with PI-RADS assessment category 4 in mp-MRI (T2WI, DWI, DCE) at 3 T with targeted plus systematic biopsy combined as the reference standard. The detection of prostate cancer with and without the use of DCE was compared.

Results Overall, the PCa detection rate in PI-RADS-4 patients was $62 \%(119 / 193)$ with DCE and $52 \%(101 / 193)$ without the inclusion of lesions upgraded on the basis of DCE. $48 \%$ (92/ 193) had clinically significant PCa (csPCa; Gleason score $\geq 3+$ $4=7)$ and $40 \%$ (78/193) without use of DCE. 38 of the 193 patients $(20 \%)$ had peripheral lesions upgraded from PI-RADS category 3 to an overall PI-RADS category 4 due to focal positive DCE findings. Of these 38 patients, 18 had PCa including 14 with csPCa. Thus, $15 \%$ (18/119) of the patients with PCa and $15 \%(14 / 92)$ of the patients with csPCa were detected only based on additional DCE information.

Conclusion DCE prevents underestimation and misclassification of a significant number of cases of peripheral csPCa and might improve detection rates in PI-RADS-4 patients. The cur- rent PI-RADS decision rules regarding upgrading PI-RADS-3 lesions to category 4 due to positive DCE imaging are useful for PCa detection.

\section{Key points:}

- Positive peripheral DCE upgraded $20 \%$ of patients in PI-RADS category 4 from category 3.

- Clinically significant PCa was found in almost $40 \%$ of upgraded, peripheral PIRADS-3-lesions.

- $15 \%$ of all csPCa in PI-RADS-4-patients was detected in DCE-upgraded lesions.

- In 7\% of all PI-RADS-4-cases csPCa would had been underestimated without DCE upgrade.

\section{Citation Format}

- Ullrich T, Quentin M, Arsov C et al. Value of Dynamic Contrast-Enhanced (DCE) MR Imaging in Peripheral Lesions in PI-RADS-4 Patients. Fortschr Röntgenstr 2020; 192: 441-447

\section{Introduction}

Magnetic resonance imaging (MRI) has become well-established and integrated in many aspects of prostate cancer (PCa) diagnostics [1, 2]. It is able to overcome the well-known limitations of PSA screening and systematic transrectal ultrasound-guided (TRUS) biopsy with respect to missing clinically significant PCa (csPCa) on the one hand and over-detecting low-grade cancer on the other hand [3]. However, the use of dynamic contrast-enhanced imaging (DCE) as an integral part of multiparametric (mp-) MRI of the prostate in addition to T2-weighted imaging (T2WI) and diffusion-weighted imaging (DWI) has been discussed controversially in the recent literature. Several authors report limited value $[4,5]$ of contrast media application and suggest a biparametric (bp) approach (T2WI, DWI), taking into account the general attempt to minimize costs and measuring time as well as to avoid potential adverse effects like allergic reactions. A recent meta-analysis of 33 single studies revealed high diagnostic accuracy for bp-MRI with respect to the detection of PCa [6]. In the head-tohead comparison, however, mp-MRI (including DCE images) showed a significantly higher pooled sensitivity $(0.85 ; 95 \% \mathrm{Cl}$, $0.78-0.93)$ compared to bp-MRI $(0.80 ; 95 \% \mathrm{CI}, 0.71-0.90)$ $(p=0.01)$, while the specificity did not differ significantly. Other studies also revealed substantial benefits from adding DCE sequences to the MRI protocol in PCa detection especially in the peripheral zone (PZ) [7-9]. In the revised PI-RADS handbook (Prostate Imaging - Reporting and Data System: 2015, Version 2) [10], which constitutes a fundamental guideline for the standardization of mp-MRI protocols and MRI reporting, DCE was assigned a minor role for final PI-RADS classification with a binary evaluation compared to a qualitative assessment in the first version of PI-RADS. Currently, DCE is particularly used to upgrade peripheral PI-RADS category 3 lesions to PI-RADS category 4 if en- hancement is focal, earlier or contemporaneous with enhancement of adjacent normal prostatic tissue. PI-RADS-5 lesions are by definition larger and therefore often more obvious compared to PI-RADS-4 lesions. Thus, DCE might be of special advantage in PI-RADS category 4. However, the diagnostic value of contrast media application in prostate MRI remains subject of the current debate and it has to be shown how many additional cases of PCa are found in upgraded PI-RADS-4 lesions. Therefore, this study strives to evaluate the differences in PCa detection with or without DCE imaging in a large cohort of patients assigned to PIRADS category 4 with targeted MRI/US fusion-guided plus systematic 12-core TRUS-guided biopsy as a reference standard.

\section{Material and Methods}

\section{Study population and design}

Consecutive patients with clinical suspicion of PCa (elevated PSA values or positive digital rectal examination) assigned to PI-RADS category 4 in standardized mp-MRI and subsequent targeted MRI/ US fusion-guided (FUS-GB) plus systematic 12-core transrectal ultrasound-guided (TRUS-GB) biopsy between January 2015 and September 2017 were included in this retrospective single-center cohort study. The patient population was previously evaluated regarding the clinical management of negatively biopsied PI-RADS4 patients [11]. The study was approved by the local Independent Ethics Committee (IEC) (Medical Faculty, University Dusseldorf) and written informed consent was obtained from all subjects.

\section{Study endpoints}

The primary endpoint was PCa detection in patients with PI-RADS category 4 upgraded from PI-RADS category 3 due to positive 
DCE findings according to the current PI-RADS decision rules. Secondary endpoints were overall PCa detection, Gleason distribution and comparison of clinical data of patients with or without PIRADS upgrade due to positive DCE.

\section{Imaging}

MRI examinations were performed on 3 T MRI scanners (Magnetom TIM Trio ${ }^{\mathrm{TM}}$, Prisma ${ }^{\mathrm{TM}}$ or Skyra ${ }^{\mathrm{TM}}$; Siemens Healthcare $\mathrm{GmbH}$, Germany) with a phased-array-surface coil according to national and international recommendations [10, 12]. The MRI protocol contained axial, sagittal and coronal T2-weighted turbo spinecho sequences (FOV $130 \mathrm{~mm}$, voxel size $0.5 \times 0.5 \times 3.0 \mathrm{~mm}$ ) and axial T1-weighted turbo spin-echo images (FOV $350 \mathrm{~mm}$, voxel size $0.6 \times 0.6 \times 5.0 \mathrm{~mm}$ ) for anatomic imaging. DWI and DCE imaging was conducted for functional assessment. DWI was acquired with a single-shot spin-echo echo-planar sequence (FOV $200 \mathrm{~mm}$, voxel size $1.5 \times 1.5 \times 3.0 \mathrm{~mm}$ ) with b-values $0,500,1000 \mathrm{~s} / \mathrm{mm}^{2}$ plus calculated $b$-values of $1600 \mathrm{~s} / \mathrm{mm}^{2}$. Apparent diffusion coefficient $(A D C)$ parameter maps were calculated using the standard monoexponential model. For DCE imaging fat-suppressed T1 vibe sequences (TR $3.87 \mathrm{~ms}$, TE $1.46 \mathrm{~ms}$, FOV $200 \mathrm{~mm}$, voxel size $0.8 \times 0.8 \times 3.0 \mathrm{~mm}$ ) were applied with a total imaging time of $3.06 \mathrm{~min}$ and a temporal resolution < $8 \mathrm{~s}[13,14]$. Gadoteridol (ProHance ${ }^{\circledR}$, Bracco) was used as the contrast media in a weightadapted standard dose $(0.2 \mathrm{mmol} / \mathrm{kg}$ body weight) with an injection rate of $3 \mathrm{ml} / \mathrm{s}$ and subsequent $50 \mathrm{ml}$ of normal saline. All patients received preliminary butylscopolamine (20 mg Buscopan ${ }^{\circledR}$, Boehringer Ingelheim Pharma) to suppress bowel peristalsis [15].

\section{Image interpretation and data analysis}

Image interpretation was done by two radiologists (T.U., L.S.) with 4 and 9 years of experience reading prostate MRI. Assessment was performed according to PI-RADS v2. Prostate volume was measured by software (DynaCAD, Invivo, Gainsville, USA) and PSA density (PSAD) was calculated by dividing PSA blood levels by prostate volume. Color-coded, parametric maps for additional DCE image evaluation were derived using the same software. Besides standard PI-RADS assessment, PCa detection rates were calculated separately for lesions upgraded from PZ PI-RADS-3 lesions to PI-RADS category 4 due to a positive, suspicious DCE imaging appearance (focal and early/contemporaneous enhancement compared to adjacent normal prostatic tissue) according to the current PIRADS decision rules. Demographic data (age), clinical data (PSA, PSAD), MRI (prostate volume, longest lesion diameter), and biopsy data were evaluated and described according to the Standards of Reporting for MRI-targeted Biopsy Studies (START) [16].

\section{Biopsy and histopathology}

Transrectal targeted FUS-GB (two targeted cores from each lesion) and subsequent systematic 12-core TRUS-GB were conducted on an MRI/US fusion-guided biopsy system with elastic registration (Urostation, Koelis or UroNAV, Invivo) using an 18G fully automatic biopsy gun (Bard Medical). All biopsies were performed by two experienced urologists (C.A., A.H.) with 8 and 6 years of experience in MR-targeted transrectal prostate biopsy, respectively. Gleason evaluation was conducted according to the recom- mendations of the International Society of Urological Pathology (ISUP). The Gleason score and cancer involvement were recorded for each biopsy core. CsPCa was defined as Gleason score $\geq 3+$ $4=7$ (ISUP class $\geq 2$ ) [17].

\section{Statistical analysis}

Statistical analyses were performed using IBM SPSS ${ }^{\circledR}$ Statistics (Version 21, IBM Deutschland $\mathrm{GmbH}$ ). Data are expressed as mean \pm SD and median + IQR. Descriptive statistics were used to present patient characteristics.

\section{Results}

\section{Patients}

193 patients with PI-RADS category 4 in mp-MRI and subsequent targeted MRI/US fusion-guided (FUS-GB) plus systematic 12-core transrectal ultrasound-guided (TRUS-GB) biopsy were analyzed. 38 patients (20\%) showed peripheral PI-RADS-4 lesions that were upgraded by positive DCE. The baseline characteristics did not differ significantly between patients with PI-RADS upgrade to category 4 and patients without upgraded lesions ( $\vee$ Table 1 ).

\section{Cancer detection and PI-RADS upgrade}

PCa was detected in 119 of 193 subjects (62\%; 131 PCa-positive lesions) including 92 (48\%) cases of csPCa with GS $\geq 3+4=7.18$ of 38 patients with upgraded PI-RADS-3 lesions had PCa including 14 cases of csPCA. Thus, $15 \%$ (18/119) of all patients with cancer and $15 \%(14 / 92)$ of patients with csPCa were detected based on DCE information. Without lesions upgraded based on DCE, the overall PCa detection rate would have been $52 \%$ (101/193) for all patients with prostate cancer. The csPCa detection rate would have been $40 \%$ (78/193). Detailed Gleason scores are illustrated in $>$ Table 2. On a lesion basis, 92 of a total of 326 PI-RADS-4 lesions were upgraded due to focal, positive DCE ( $>$ Table 3 ). Of the upgraded lesions, 38 (41\%) were positive for PCa including 30 lesions with csPCa (33\%). An example of an upgraded PZ PCa lesion is shown in $>$ Fig. 1. The median PSAD of patients with upgraded lesions and subsequently verified PCa (0.20; IQR: 0.18-0.22) did not differ significantly from the PSAD of patients with upgraded lesions without PCa (0.16; IQR: 0.11-0.17; $\mathrm{P}=0.28)$.

\section{Discussion}

This study demonstrates the importance of dynamic contrast-enhanced imaging (DCE) when upgrading peripheral PI-RADS-3 lesions to PI-RADS category 4 in order not to miss clinically significant tumors. $33 \%$ of the peripheral higher-grade PCa lesions would have been underestimated and misclassified as PI-RADS 3 without the help of DCE in this study. The current debate on whether DCE may be omitted in favor of a bi-parametric (T2WI, DWI) MRI protocol, even in the setting of primary tumor detection should, therefore, be held with caution.

In this study we demonstrate high PCa detection rates in PI-RADS- 4 patients of $62 \%$ including csPCa rates of $48 \%$ which is 
- Table 1 Baseline characteristics of all patients and of patients with and without lesions initially categorized as PI-RADS 3 in DWI and upgraded to PI-RADS category 4 due to positive DCE.

- Tab. 1 Baseline-Charakteristiken aller Patienten und der Patienten mit und ohne Läsionen, die durch fokale Kontrastmittelanreicherung von PI-RADS-Kategorie 3 auf eine Gesamtkategorie 4 aufgewertet wurden.

\begin{tabular}{|c|c|c|c|c|}
\hline & & all patients $(n=193)$ & $\begin{array}{l}\text { patients with upgrade based } \\
\text { on DCE }(n=38)\end{array}$ & $\begin{array}{l}\text { patients } w / 0 \text { upgrade based } \\
\text { on DCE }(n=155)\end{array}$ \\
\hline age $[y]$ & mean $\pm S D$ & $65 \pm 9$ & $63 \pm 9$ & $65 \pm 9$ \\
\hline PSA [ng/ml] & median (IQR) & $7.6(5.6-11)$ & $6.9(5.2-10)$ & $7.7(5.9-10)$ \\
\hline PSA density $[\mathrm{ng} / \mathrm{ml} / \mathrm{ml}]$ & median (IQR) & $0.17(0.11-0.24)$ & $0.18(0.11-0.25)$ & $0.17(0.11-0.24)$ \\
\hline prostate volume [ml] & median (IQR) & $45(31-67)$ & $38(26-57)$ & $47(32-64)$ \\
\hline
\end{tabular}

Note: $\mathrm{PSA}=$ prostate specific antigen; $\mathrm{SD}=$ standard deviation; $\mathrm{IQR}=$ interquartile range; $\mathrm{w} / \mathrm{o}=$ without.

- Table 2 PCa detection and Gleason score distribution of all patients and of patients with and without lesions initially categorized as PI-RADS 3 in DWI and upgraded to PI-RADS category 4 due to positive DCE.

- Tab. 2 Prostatakarzinomdetektion und Gleason-Score-Verteilung aller Patienten und der Patienten mit und ohne Läsionen, die durch auffällige, fokale Kontrastmittelanreicherung von PI-RADS-Kategorie 3 auf eine Gesamtkategorie 4 aufgewertet wurden.

\begin{tabular}{|l|l|l|l|}
\hline & $\begin{array}{l}\text { all patients } \\
\text { (n= 193) }\end{array}$ & $\begin{array}{l}\text { patients } \\
\text { with up- } \\
\text { grade based } \\
\text { on DCE } \\
\text { (n=38) }\end{array}$ & $\begin{array}{l}\text { patients w/o } \\
\text { upgrade } \\
\text { based on } \\
\text { DCE (n= 155) }\end{array}$ \\
\hline any cancer [\% (n)] & $62(119)$ & $47(18)$ & $65(101)$ \\
\hline GS $\geq 7$ [\% (n)] & $48(92)$ & $37(14)$ & $50(78)$ \\
\hline $\begin{array}{l}\text { PCa detection w/o } \\
\text { upgrade based on } \\
\text { DCE [\% (n)] }\end{array}$ & $52(101)$ & - & - \\
\hline $\begin{array}{l}\text { GS } \geq 7 \text { PCa detection } \\
\text { w/o upgrade based } \\
\text { on DCE [\% (n)] }\end{array}$ & $40(78)$ & - & - \\
\hline gleason score (GS) distribution [\% (n)] & & \\
\hline GS 3 +3=6 & $14(27)$ & $11(4)$ & $15(23)$ \\
\hline GS 3+4=7a & $26(50)$ & $21(8)$ & $27(42)$ \\
\hline GS 4+3=7b & $11(21)$ & $8(3)$ & $12(18)$ \\
\hline GS 8 & $6.7(13)$ & $8(3)$ & $6(10)$ \\
\hline GS 9 & $2.6(5)$ & 0 & $3(5)$ \\
\hline GS 10 & $0.5(1)$ & 0 & $1(1)$ \\
\hline
\end{tabular}

Note: $\mathrm{GS}=$ Gleason score; $\mathrm{PCa}$ = prostate cancer; $\mathrm{DCE}=$ dynamic contrast-enhanced imaging; $\mathrm{w} / \mathrm{O}=$ without.

in line with the findings by Venderink et al. [18] who revealed PCa in $60 \%$ and csPCa in $34 \%$. Every csPCa with GS $\geq 7$ could reliably be detected on mp-MRI in this study. However, the reported PCa detection in PI-RADS category 4 varies greatly in the literature down
- Table 3 PCa detection in PZ lesions with initial PI-RADS category 3 in DWI and upgrade to PI-RADS category 4 due to positive, suspicious DCE.

- Tab. 3 Prostatakarzinomdetektion in peripheren Läsionen mit initialer PI-RADS-Kategorie 3 in der DWI und Aufwertung auf Kategorie 4 durch auffällige, fokale Kontrastmittelanreicherung.

\begin{tabular}{|l|l|}
\hline & $\begin{array}{l}\text { PZ Lesions upgraded based on } \\
\text { DCE }\end{array}$ \\
\hline $\begin{array}{l}\text { PCa detection in upgraded } \\
\text { lesions [\% (n)] }\end{array}$ & $41(38 / 92)$ \\
\hline $\begin{array}{l}\text { GS } \geq 7 \text { PCa } \\
\text { detection in upgraded } \\
\text { lesions }\end{array}$ [\% (n) $]$ & $33(30 / 92)$ \\
\hline
\end{tabular}

Note: $\mathrm{PZ}$ = peripheral zone; $\mathrm{PCa}=$ prostate cancer; $\mathrm{GS}=$ Gleason score; $\mathrm{DCE}=$ dynamic contrast-enhanced imaging.

to $39 \%$ by Mehralivand et al. [19]. Differences in image acquisition, experience of the reader, and subjective interpretation criteria may be responsible for these diverse results. Apart from this, PI-RADS-4 lesions are generally prone to sampling errors due to their smaller size compared to PI-RADS-5 lesions. $15 \%$ of all detected $\mathrm{PCa}$ cases in our patient cohort were detected in peripheral lesions upgraded from DWI PI-RADS category 3 to category 4 due to positive DCE findings according to the current PI-RADS decision rules. This corresponds to the results from Rosenkrantz et al. [20] who revealed a csPCa rate of up to $33 \%$ in upgraded $\mathrm{PZ}$ lesions and to the findings by Greer et al. [7] who reported improved cancer detection for PI-RADS-3 and 4 lesions in the PZ when DCE imaging is performed.

In recent times the need for DCE as part of the standard prostate MRI protocol has been discussed controversially based on the desire to reduce scanning time and avoid contrast material administration [7, 21, 22]. Some authors suggest bp-MRI in combination with the PSAD level as the diagnostic tool to decide whether a patient needs to be biopsied [5] since the histopatholo- 

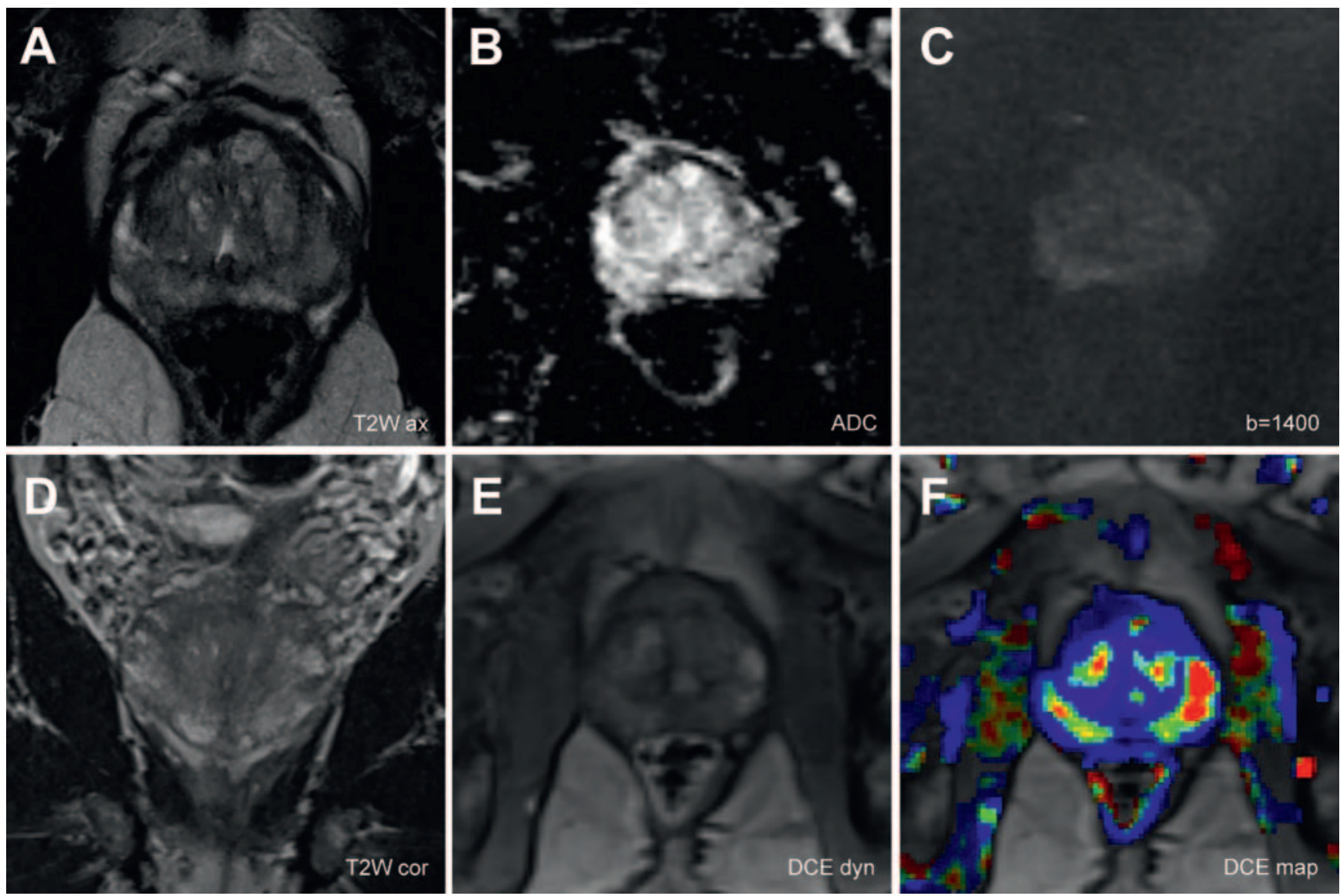

- Fig. 1 Example of a peripheral lesion upgraded from DWI PI-RADS category 3 to an overall category PI-RADS 4 due to positive DCE findings. Case: 65 -year-old man, PSA $9.5 \mathrm{ng} / \mathrm{ml}$ - Lesion with diffuse and partly focal hypointensity in T2WI A, D without clear focal diffusion restriction B, C but focal early contrast enhancement E, F in mid left peripheral zone. Histopathology revealed 6 of 18 positive cores (GS $3+4=7$ a; PZpl mid left; up to $70 \%$ in the corresponding targeted core).

- Abb. 1 Beispiel einer peripheren Läsion, die durch eine auffällige fokale Kontrastmittelanreicherung von der PI-RADS-Kategorie 3 auf eine Gesamtkategorie 4 aufgewertet wurde. Fall: 65 Jahre alter Mann, PSA 9,5 ng/ml; Indexläsion mit diffuser teilweise fokaler Hypointensität in der T2Wichtung A, D ohne eindeutig fokale Diffusionsrestriktion B, C, jedoch mit fokaler Kontrastmittelanreicherung E, $\mathbf{F}$ in der mittleren linken peripheren Zone. Histopathologische Evaluation ergab 6 von 18 positiven Stanzen (GS 3+4 =7a; PZpl mit links; bis zu $70 \%$ in den gezielten Stanzen).

gic evaluation is still the ultimate gold standard to detect PCa [23]. However, in our study one third of the csPCa lesions would have been misclassified as PI-RADS 3 without the help of DCE and might not have been detected under the assumption that PI-RADS-3 lesions are not biopsied. At our institution, PI-RADS-3 lesions do not get biopsied on a regular basis due to the overall low cancer detection rate in this category and to avoid overdiagnosis of low-risk cancers with the potential for unnecessary adverse effects. Previous studies have revealed that the overall PCa detection rate in PI-RADS category 3 is low and can be managed by follow-up mp-MRI [24] in order to avoid unnecessary biopsies. Fewer biopsies are not only more convenient for the patient but also minimize the risk of complications such as infection [25]. Other authors propose administering contrast media only in doubtful cases (PI-RADS 3) either in a second session or in the same session with the radiologist immediately assessing the MRI images [4]. This requires a highly organized clinical setting. Also, very few patients will profit from such an approach since primarily higher risk patients are referred to mp-MRI and a majority have suspicious lesions.

Besides upgrading PI-RADS-3 to PI-RADS-4 lesions, DCE imaging adds sensitivity by assisting in index lesion selection. Some lesions that might not have attracted attention in the other sequences become suspicious through focal, intense, and early enhancement, considering especially that DWI is more prone to artifacts and can be falsely unremarkable [26]. In our study two patients with upgraded PCa lesions with a Gleason score of 6 showed DWI that was not qualitatively sufficient due to artifacts.

Furthermore, DCE also adds specificity by reducing the number of false-positive results as non-vascularized lesions might be excluded and not get biopsied. DCE imaging is also helpful in tumor detection in the anterior fibromuscular stroma or in the central zone. These aspects were, however, not analyzed in this study. The median PSAD of patients with upgraded PI-RADS-3 lesions and subsequently verified PCa was higher compared to upgraded patients without PCa. However, the effect was not statisti- 
cally relevant suggesting that PSAD can give a helpful hint about the PCa risk but cannot be used as a definite predictor.

A potential limitation of this study is the fact that we only included patients with PI-RADS category 4. Thus, PI-RADS 3 lesions with a negative DCE were not assessed, so that there is no data to calculate specificity. We also did not investigate how many cancers would have been missed in complete absence of contrast-enhanced sequences as the basis for calculation of sensitivity. However, several previous studies revealed that a relevant proportion of cases of csPCa will be missed if DCE sequences are not used [27, 28 ], provided that optimum acquisition and expert evaluation are guaranteed. Unfortunately, DCE images are often not acquired according to current guidelines (MRI parameters, contrast media application), which in turn leads to similar PCa detection rates compared to bp-MRI and complicates the debate. As the study was designed to assess PCa detection in upgraded PZ lesions, other locations and other clinical or MRI morphological data were not evaluated.

In conclusion, DCE prevents the misclassification of a significant number of cases of clinically significant peripheral PCa that would have been underestimated in a bi-parametric protocol. Therefore, DCE plays an important role in primary tumor detection. The current PI-RADS decision rules regarding upgrading PIRADS-3 lesions to category 4 due to positive DCE imaging are useful. Further studies prospectively investigating a head-to-head comparison of PCa detection with or without DCE imaging are warranted.

\section{CLINICAL RELEVANCE}

- Contrast media application can support the detection of clinically significant prostate cancer in mp-MRI of the prostate.

- Dynamic contrast-enhanced sequences might especially be helpful in unclear cases in the setting of primary (early) tumor detection.

- If dynamic contrast-enhanced imaging is used, peripheral PI-RADS-3 lesions might get upgraded to PI-RADS category 4 in $20 \%$ of patients.

\section{Conflict of Interest}

The authors declare that they have no conflict of interest.

\section{References}

[1] Barentsz J, de Rooij M, Villeirs G et al. Prostate Imaging-Reporting and Data System Version 2 and the Implementation of High-quality Prostate Magnetic Resonance Imaging. Eur Urol 2017; 72: 189-191

[2] Woo S, Suh CH, Kim SY et al. Diagnostic Performance of Prostate Imaging Reporting and Data System Version 2 for Detection of Prostate Cancer: A Systematic Review and Diagnostic Meta-analysis. Eur Urol 2017; 72: 177188

[3] Siddiqui MM, Rais-Bahrami S, Turkbey B et al. Comparison of MR/ultrasound fusion-guided biopsy with ultrasound-guided biopsy for the diagnosis of prostate cancer. JAMA 2015; 313: 390-397
[4] De Visschere P, Lumen N, Ost P et al. Dynamic contrast-enhanced imaging has limited added value over T2-weighted imaging and diffusionweighted imaging when using PI-RADSv2 for diagnosis of clinically significant prostate cancer in patients with elevated PSA. Clin Radiol 2017; 72: $23-32$

[5] Fascelli M, Rais-Bahrami S, Sankineni S et al. Combined Biparametric Prostate Magnetic Resonance Imaging and Prostate-specific Antigen in the Detection of Prostate Cancer: A Validation Study in a Biopsy-naive Patient Population. Urology 2016; 88: 125-134

[6] Niu XK, Chen XH, Chen ZF et al. Diagnostic Performance of Biparametric MRI for Detection of Prostate Cancer: A Systematic Review and MetaAnalysis. Am J Roentgenol 2018; 211: 369-378

[7] Greer MD, Shih JH, Lay N et al. Validation of the Dominant Sequence Paradigm and Role of Dynamic Contrast-enhanced Imaging in PI-RADS Version 2. Radiology 2017; 285: 859-869

[8] Delongchamps NB, Beuvon F, Eiss D et al. Multiparametric MRI is helpful to predict tumor focality, stage, and size in patients diagnosed with unilateral low-risk prostate cancer. Prostate Cancer Prostatic Dis 2011; 14: 232-237

[9] Tamada T, Sone T, Higashi H et al. Prostate cancer detection in patients with total serum prostate-specific antigen levels of $4-20 \mathrm{ng} / \mathrm{mL}$ : diagnostic efficacy of diffusion-weighted imaging, dynamic contrast-enhanced MRI, and T2-weighted imaging. Am J Roentgenol 2011; 197 : 664-670

[10] Weinreb JC, Barentsz JO, Choyke PL et al. PI-RADS Prostate Imaging Reporting and Data System: 2015, Version 2. Eur Urol 2016; 69: 16-40

[11] Ullrich T, Arsov C, Quentin M et al. Analysis of PI-RADS 4 cases: Management recommendations for negatively biopsied patients. Eur J Radiol 2019; 113: 1-6

[12] Franiel T, Quentin M, Mueller-Lisse UG et al. MRI of the Prostate: Recommendations on Patient Preparation and Scanning Protocol. RöFo 2017; 189: 21-28

[13] Schimmöller L, Blondin D, Arsov C et al. MRI-Guided In-Bore Biopsy: Differences Between Prostate Cancer Detection and Localization in Primary and Secondary Biopsy Settings. AJR 2016; 206: 92-99

[14] Arsov C, Rabenalt R, Blondin D et al. Prospective randomized trial comparing MR-guided in-bore versus MRI/ultrasound fusion and TRUS-guided prostate biopsy in patients with prior negative biopsies. Eur Urol 2015; 68: 713-720

[15] Ullrich T, Quentin M, Schmaltz AK et al. Hyoscine butylbromide significantly decreases motion artefacts and allows better delineation of anatomic structures in mp-MRT of the prostate. Eur Radiol 2018; 28: 17-23

[16] Moore CM, Kasivisvanathan V, Eggener S et al. START Consortium Standards of reporting for MRI-targeted biopsy studies (START) of the prostate: recommendations from an International Working Group. Eur Urol 2013; 64: 544-552

[17] Epstein JI, Egevad L, Amin MB et al. The 2014 International Society of Urological Pathology (ISUP) Consensus Conference on Gleason Grading of Prostatic Carcinoma: Definition of Grading Patterns and Proposal for a New Grading System. Am J Surg Pathol 2016; 40: 244-252

[18] Venderink W, van Luijtelaar A, Bomers JG et al. Results of Targeted Biopsy in Men with Magnetic Resonance Imaging Lesions Classified Equivocal, Likely or Highly Likely to Be Clinically Significant Prostate Cancer. Eur Urol 2017. doi:10.1016/j.eururo.2017.02.021 [Epub ahead of print]

[19] Mehralivand S, Bednarova S, Shih JH et al. Prospective Evaluation of PI-RADS Version 2 Using the International Society of Urological Pathology Prostate Cancer Grade Group System. J Urol 2017; 198: 583-590

[20] Rosenkrantz AB, Babb JS, Taneja SS et al. Proposed Adjustments to PI-RADS Version 2 Decision Rules: Impact on Prostate Cancer Detection. Radiology 2017; 283: 119-129 
[21] Chen Z, Zheng Y, ji G et al. Accuracy of dynamic contrast-enhanced magnetic resonance imaging in the diagnosis of prostate cancer: systematic review and meta-analysis. Oncotarget 2017; 17: 77975-77989

[22] Vargas HA, Hötker AM, Goldman DA et al. Updated prostate imaging reporting and data system (PIRADS V2) recommendations for the detection of clinically significant prostate cancer using multiparametric MRI: critical evaluation using whole-mount pathology as standard of reference. Eur Radiol 2016; 26: 1606-1612

[23] Scialpi M, Falcone G, Scialpi P et al. Biparametric MRI: a further improvement to PIRADS 2.0? Diagn Interv Radiol 2016; 22: 297-298

[24] Ullrich T, Quentin M, Arsov C et al. Risk stratification of Equivocal PIRADS lesions in mp-MRI of the prostate. J Urol 2018; 199: 691-698
[25] Walker JT, Singla N, Roehrborn CG. Reducing Infectious Complications Following Transrectal Ultrasound-guided Prostate Biopsy: A Systematic Review. Rev Urol 2016; 18: 73-89

[26] Mazaheri Y, Vargas A, Nyman G et al. Image Artifacts on Prostate Diffusion-weighted Magnetic Resonance Imaging: Trade-offs at 1.5 Tesla and 3.0 Tesla. Acad Radiol 2013; 20: 1041-1047

[27] Puech P, Sufana-lancu A, Renard B et al. Prostate MRI: can we do without DCE sequences in 2013? Diagn Interv Imaging 2013; 12: 1299-1311

[28] Benndorf M, Waibel L, Krönig M et al. Peripheral zone lesions of intermediary risk in multiparametric prostate MRI: Frequency and validation of the PI-RADSV2 risk stratification algorithm based on focal contrast enhancement. Eur J Radiol 2018; 99: 62-67 